\title{
Signaling network of OSW-1-induced apoptosis and necroptosis in hepatocellular carcinoma
}

\author{
JICHUN JIN, XINGLIN JIN, CHANGSHI QIAN, YANG RUAN and HAO JIANG
}

Department of Hepatopancreatobiliary Surgery, Affiliated Hospital of Yanbian University, Yanji, Jilin 133000, P.R. China

Received November 30, 2012; Accepted February 18, 2013

DOI: $10.3892 / \mathrm{mmr} .2013 .1366$

\begin{abstract}
The compound $3 \beta, 16 \beta, 17 \alpha$-trihydroxycholest-5 -en-22-one 16-O-(2-O-4-methoxybenzoyl- $\beta$-D-xylopyranosyl)-(1-3)-(2-O-acetyl- $\alpha-\mathrm{L}$-arabinopyranoside (OSW-1) is a member of the cholestane saponin family that was created in the bulbs of Ornithogalum saudersiae. OSW-1 has previously been shown as cytotoxic against numerous types of malignant cells, however, its antitumoral mechanisms remain unclear. The present study aimed to examine the potential changes in the gene expression of a hepatocellular carcinoma (HCC) cell line (Hep3B) incubated with OSW-1 in vitro. The results showed that OSW-1 inhibited tumors through invasiveness, angiogenesis, cell polarity and cell adhesion (as shown by Roche NimbleGen gene expression analysis), in addition to inducing apoptosis through the mitochondrial pathway. This affected the expression of a number of core genes in a number of signaling pathways, including WNT, MAPK, VEGF and P53. To the best of our knowledge, the present study is the first to report that OSW-1, as a molecular compound, induces necroptotic death in hepatocellular carcinoma (HCC).
\end{abstract}

\section{Introduction}

$3 \beta, 16 \beta, 17 \alpha$-trihydroxycholest-5-en-22-one 16-O-(2-O-4methoxybenzoyl- $\beta$-D-xylopyranosyl)- $(1 \rightarrow 3)-(2-\mathrm{O}$-acetyl- $\alpha$-Larabinopyranoside) (OSW-1) is located in the bulbs of Ornithogalum saudersiae (1) and its anticancer effect is 10-100 times greater than that of doxorubicin, camptothecin or paclitaxel (2). Non-malignant cells are significantly less sensitive to OSW-1 than cancer cell lines, with concentrations that cause a $50 \%$ loss of cell viability of 40-150-fold greater than that observed in malignant cells. More significantly, OSW-1 can lead to a loss of mitochondrial transmembrane

Correspondence to: Professor Xinglin Jin, Department of Hepatopancreatobiliary Surgery, Affiliated Hospital of Yanbian University, Yanji, Jilin 133000, P.R. China

E-mail: xljin@ybu.edu.cn

Key words: OSW-1, hepatocellular carcinoma, signaling pathways, gene, necroptotic death potential, an increase in cytosolic calcium and the activation of calcium-dependent apoptosis in human leukemia and pancreatic cancer cells (3). However, the method by which OSW-1 exerts its anticancer activity is complex and the exact mechanisms responsible for such selectivity remain unclear. To investigate the mechanism of this unnatural anticancer activity, gene expression analysis was used to examine the potential changes in the gene expression of a hepatocellular carcinoma (HCC) cell line (Hep3B) that had been incubated with OSW-1 in vitro. A network extension of the signaling pathways that participate in apoptosis and necroptosis mediated by OSW-1 was then created.

\section{Materials and methods}

Cell cultures. Hep3B was obtained from the Chinese Academy of Sciences Cell Bank and the cell line was maintained in Dulbecco's modified Eagle's medium (DMEM; Invitrogen, Carlsbad, CA, USA) supplemented with $10 \%$ fetal bovine serum (Gibco Life Technologies, Carlsbad, CA, USA). A monoclonal cell line was acquired using a limiting dilution assay (LDA) and maintained in DMEM with $20 \%$ fetal bovine serum. A humidified incubator was set at $37^{\circ} \mathrm{C}$ with $5 \% \mathrm{CO}_{2}$. The Hep3B monoclonal cell line was treated with $200 \mathrm{ng} / \mathrm{ml}$ OSW-1 for $24 \mathrm{~h}$.

RNA isolation. A total of $7 \times 10^{6}$ monoclonal cells were used for total RNA isolation. The total RNA was isolated using a TRIzol reagent according to the manufacturer's instructions (Invitrogen, Hong Kong, China). The RNA concentration was determined by measuring the absorbance at $260 \mathrm{~nm}$ using the NanoDrop ND1000 spectrophotometer (Thermo Scientific, Waltham, MA, USA) and the purity of the RNA was estimated using the OD260/280 ratio. The RNA integrity was assessed by standard denaturing agarose gel electrophoresis, then the RNAs were used for labeling and array hybridization.

cDNA synthesis and labeling. In total, $10 \mu \mathrm{g}$ of the RNA was processed and labeled according to the standard NimbleGen instructions. Briefly, the RNA was converted into cDNA using a Superscript Double-Stranded cDNA Synthesis kit (Invitrogen). The double-stranded cDNA was random-prime labeled with Cy3 converted via an oligo-dT using a NimbleGen One-Color DNA Labeling kit subsequent to RNase cleanup and cDNA precipitation. 
Table I. Genes from the various signaling pathways that were downregulated by OSW-1.

Signaling pathway

Wnt

MAPK

VEGF
Downregulated genes

CCND1/CCND2/CSNK1E/FRAT2/FZD2/FZD9/JUN/LRP5/MYC/NFATC1/NKD2/PPP2R5B/ RAC3/TCF7L1/WNT10A/WNT10B/WNT5B/WNT7B/WNT9A ARRB1/BDNF/CD14/DUSP1/DUSP2/DUSP4/DUSP5/DUSP9/FGF11/FGF21/FGF8/FGFR1/ FGFR4/FLNB/FLNC/HSPA1A/HSPA6/IL1B/JUN/MAP2K1/MAP2K3/MAP2K6/MAP3K12/ MAP3K6/MAPK13/MAPKAPK2/MKNK2/MRAS/MYC/PAK1/PDGFB/PLA2G12A/PPP5C/ RAC3/RPS6KA1/RRAS/SRF

CASP9/MAP2K1/MAPK13/MAPKAPK2/NFATC1/NOS3/PIK3CD/PLA2G12A/PLCG2/RAC3

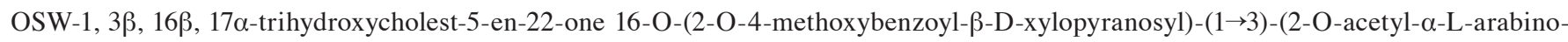
pyranoside.

Expression profiling using microarrays. The labeled cRNAs were hybridized to the NimbleGen Human Gene Expression $12 \times 135 \mathrm{~K}$ microarray using the following steps: i) Reverse transcription with the Invitrogen Superscript ds-cDNA Synthesis kit; ii) ds-cDNA labeling with the NimbleGen One-Color DNA Labeling kit; iii) array hybridization using the NimbleGen Hybridization System followed by washing with the NimbleGen Wash Buffer kit; and iv) array scanning using the Axon GenePix 4000B microarray scanner (Molecular Devices Co., Sunnyvale, CA, USA).

\section{Results}

Data analysis. After washing, the slides were scanned with an Axon GenePix 4000B scanner. The data were extracted and normalized using NimbleScan v2.5 Software. The raw signal intensities were normalized using the RMA method with NimbleScan v2.5 and the low intensity genes were filtered (genes that had at least 2 out of 2 samples with values $\geq$ the lower cut-off; 50.0 were chosen for further analysis). The quality of the gene data was assessed using box and scatter plots subsequent to filtering. The differentially expressed genes that passed fold change filtering (fold change, $\geq 2.0$ ) and the final data were used to create a heat map, then the hierarchical clustering pathway analysis was completed (Fig. 1). The box plot shown in Fig. 1 is a convenient way to quickly visualize the distributions of a dataset. It is commonly used for comparing the distributions of the intensities from all samples. After normalization, the distributions of log2-ratios among all tested samples are nearly the same. The scatterplot shown in Fig. 1 is a visualization method used for assessing the expression variation (or reproducibility) between two groups. The values of $\mathrm{X}$ and $\mathrm{Y}$ axes in the scatterplot are the normalized signal values of each sample ( $\log 2$ scaled) [or averaged normalized signal values of each group ( $\log 2$ scaled)]. The three lines are fold change lines (the fold change value given is 2.0). Genes above the top line and below the bottom line indicated more than 2.0-fold change of genes between two groups.

Pathway analysis. OSW-1 affected the expression of numerous genes in vitro. Despite the fact that OSW-1 was at a nanomolar concentration, 570 genes were downregulated and 341 genes were upregulated. Differential expression analysis of the genes

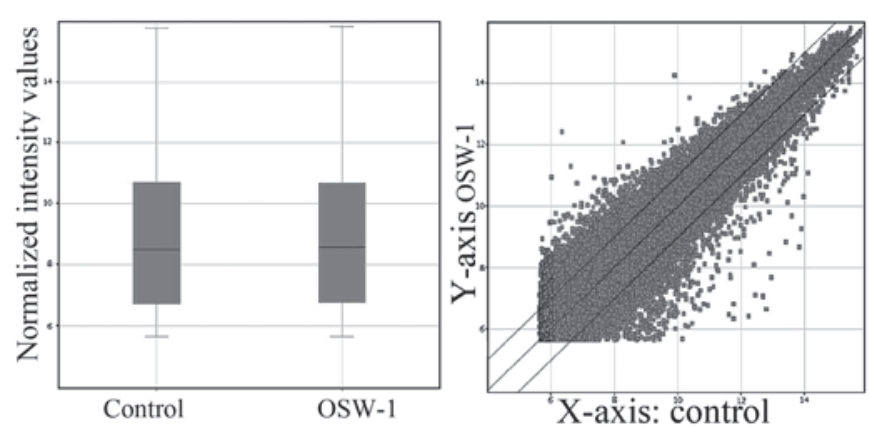

Figure 1. Boxplot visualizing the distribution of the dataset of the control and OSW-1 groups and scatterplot to assess the variation or reproducibility between the microarray chips. OSW-1, 3 $\beta, 16 \beta, 17 \alpha$-trihydroxycholest-5-en-2 2-one 16-O-(2-O-4-methoxybenzoyl- $\beta$-D-xylopyranosyl)-(1 $\rightarrow 3)-(2-\mathrm{O}$-acetyl$\alpha$-L-arabinopyranoside.

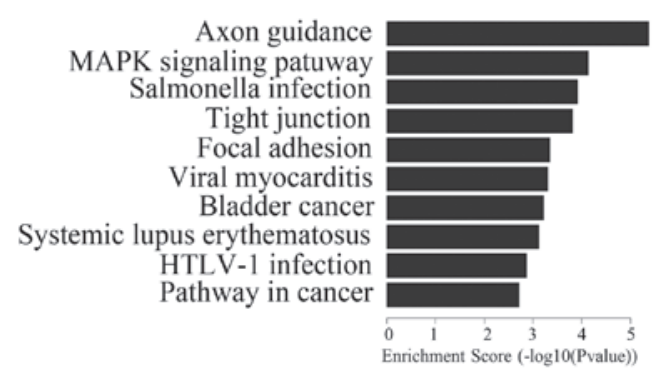

Figure 2. Enrichment analysis of the differentially expressed (DE) gene in the downregulation of the signaling pathway. The enrichment score is the enrichment score value of the PathwayID, it equals '-log10(P-value)'.

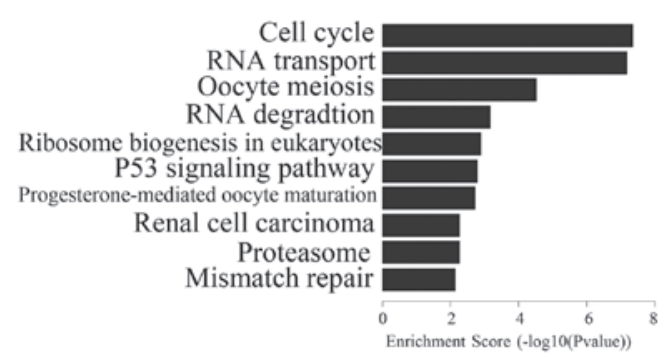

Figure 3. Enrichment analysis of the differentially expressed (DE) gene in the upregulation of the signaling pathway. The enrichment score is the enrichment score value of the PathwayID, it equals '- $\log 10$ (P-value)'. 
Table II. Genes from the various signaling pathways that were upregulated by OSW-1.

Signaling pathway

P53

Cell cycle
Upregulated genes
CASP8/CCNB1/CCNB2/CHEK1/CYCS/GTSE1/PERP/PMAIP1/PPM1D/RRM2/SERPINB5/ SIAH1/THBS1

ANAPC1/ANAPC7/BUB1/BUB3/CCNA2/CCNB1/CCNB2/CDC16/CDC23/CDC25B/CDC25C/ CDC25C/CDC6/CHEK1/CUL1/E2F2/E2F3/EP300/HDAC2/PCNA/PRKDC/PTTG1/RAD21/ SKP2/SMAD4/TGFB2/WEE1/YWHAB/YWHAQ/YWHAZ

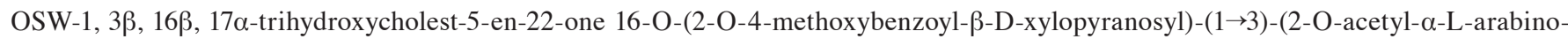
pyranoside.

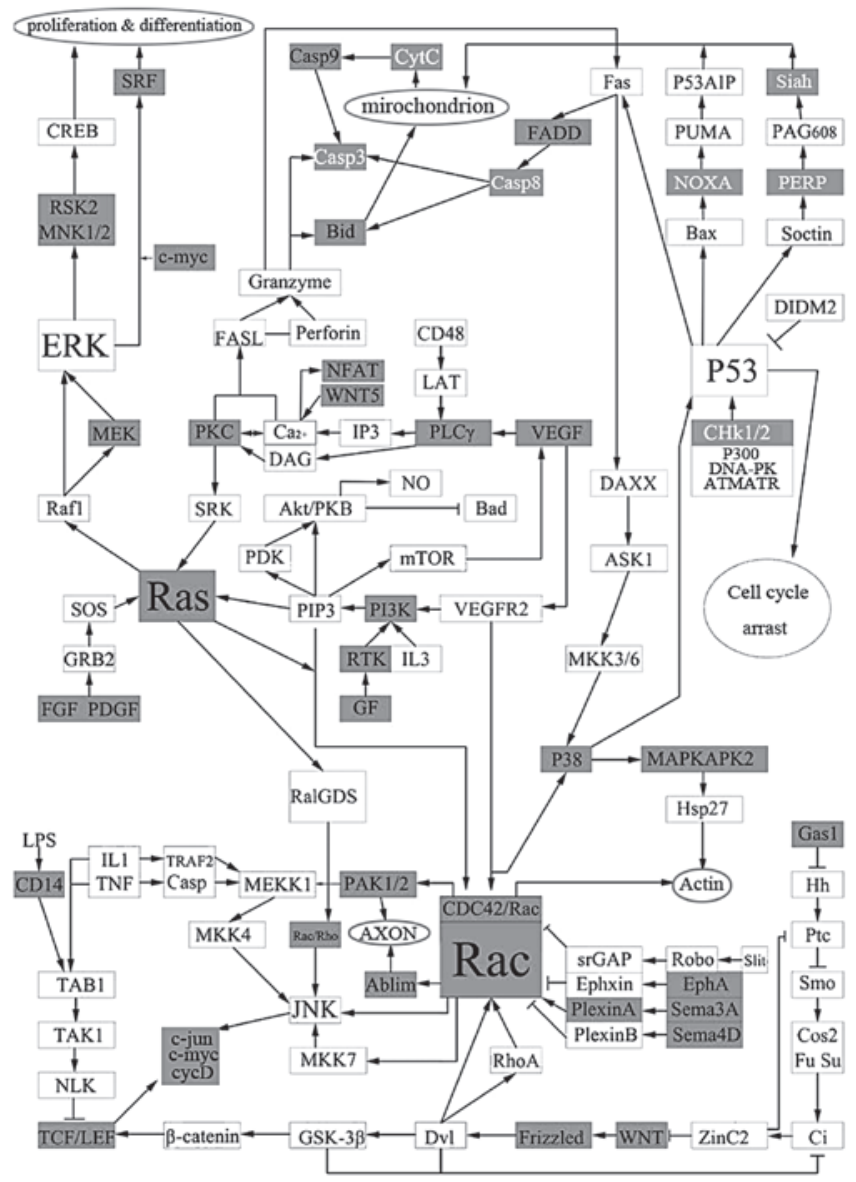

Figure 4. Network extension of the signaling pathway collated by the differentially expressed genes that were correlated with OSW-1, shown in a comprehensive pathway. The gray nodes with black words are associated with the downregulated genes and the gray nodes with the white words are associated with the upregulated genes. Only white nodes have no significance in Hep3B cells, but whether they constitute a part of the connection between parts of the pathway and OSW-1 requires further study.

was performed in the Hep3B cells that had been treated with OSW-1 and included the analysis of PDGF, Ras, c-Myc, MEK1, WNT, TCF/LEF, PI3K, caspase-8,9, FADD, CytC and Chk1/2. These genes are involved in certain pathways, including those for axon guidance, MAPK, tight junctions, WNT, the cell cycle and P53 (Figs. 2 and 3; Tables I and II). These results indicated that OSW-1 OSW-1 inhibits cell invasion, angiogenesis, cell adhesion and destruction of cell polarity.

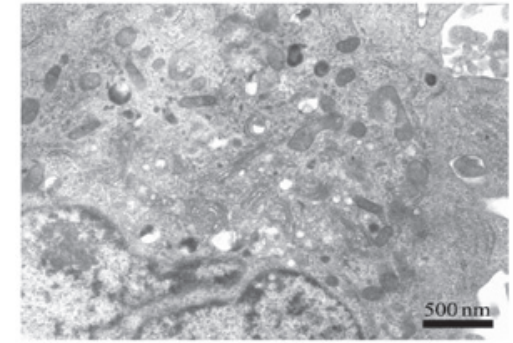

Figure 5. Hep3B cells treated with $200 \mathrm{ng} / \mathrm{nl}$ OSW-1 for $24 \mathrm{~h}$ as shown by transmission electron microscopy scanning. The images shows the following: the nucleus appeared depressed, deformed and karyopyknosis was observed; cytoplasmic vacuoles, mitochondrial swelling and mitochondrial cristae disappeared; degranulation of rough endoplasmic reticulum and sliding surface of endoplasmic reticulum cystic dilatation was observed.

\section{Discussion}

In the present study, the NimbleGen expression values correlated strongly with the TaqMan expression values, the current 'gold standard' for gene expression quantitation, demonstrating the accuracy of the data obtained through using this technology. In addition to highly reproducible and accurate expression values, the unique combination of NimbleGen high-density arrays, long oligos and flexible design abilities provides superior results. The raw signal intensities were normalized using the RMA method with NimbleScan v2.5 and the low intensity genes were filtered, followed by the differentially expressed genes that passed fold change filtering.

To investigate the role of OSW-1 on Hep3B, the differentially expressed genes that were associated with OSW-1 were collated into a comprehensive pathway (Fig. 4). Mitochondria play a critical role in mediating the apoptotic signal transduction pathway which includes mitochondrial swelling and disruption of the mitochondrial outer membrane and cristae when treated by OSW-1 (Fig. 5).

Once the classical WNT signaling pathway is activated, it may cause target gene overexpression. c-myc, cyclinD1 and c-jun have been determined as a few of the proto-oncogenes that are the target genes affecting cell fate, migration and polarity (4). These genes are all WNT family glycoproteins with conserved 22-24 Cys residues that are downregulated by OSW-1 in the WNT signaling pathway. More prominently, the Frizzled and TCF/LEF genes demonstrate the same characteristics. This suggests that OSW-1 may act on WNT 
proteins directly, with its effects visible in the cytoplasm and cell nucleus.

The ERK signaling pathway plays a key role in several steps of tumorigenesis (5). Ras activates the ERK pathway through receptor-mediated activation of the small G-protein (6). Ras is activated through the exchange of bound GDP into GTP as a membrane-bound protein. OSW-1 is able to prevent this progress specifically. It has been demonstrated that Ras/ Raf-independent activation of MEK1/2 is inhibited downstream. ERK1/2-mediated signaling plays a significant role in mediating cell survival. The activated alleles of MEK1/2 promote cell survival independently of other survival factors (7) and OSW-1 downregulates MEK1 expression. RSK, which is also downregulated by OSW-1, activates the transcription factor CREB, which promotes cell survival through transcriptional upregulation of the antiapoptotic $\mathrm{Bcl}$ family of proteins $(8,9)$. Taken together, these OSW-1-based findings and those obtained from expression profiling in the present study, may support the results of a previous study (10). The induction of apoptosis in several cell lines requires the activation of the p38 MAPK pathway (11). In the present study, OSW-1 acted on Hep3B, MKK3 MKK6 and P38, whose transcription levels were lower than the control groups without the activation of an upstream gene in the control groups. From observing the enrichment score of the MAPK signaling pathway (-Log10P-value) this pathway may be considered as one of the most significant mechanisms. In KRAS mutants and RAS/RAF wild-type tumors, RAF inhibitors activate the RAF-MEK-ERK pathway in a RAS-dependent manner, thus enhancing tumor growth in certain engraft models (m14). Non-upstream gene activation mediated by OSW-1 indicates that it may direct the conformational effects of the inhibitors on the RAF kinase domain as the RAS and mitochondrial apoptosis effects mediate RAS-GTP signaling.

Previous studies have demonstrated that the VEGF/ RTK/PI3K/AKT pathway plays a key role in regulating migration, angiogenesis, proliferation and survival $(12,13)$. Downregulation of eNOS increases the sensitivity of $\mathrm{Ca}^{2+}$ for apoptosis induced by the mitochondria. This observation may demonstrate, through a complex signaling pathway, the fact that the VEGF signaling pathway has a key role in regulating tumor angiogenesis and an auxiliary role in apoptosis of the mitochondria which is induced by OSW-1 in HCC. However, VEGF may promote tumor growth and be inhibited by other reagents (14). This may be evidence of the particular anticancer mechanisms that increase the cytosolic calcium in the mitochondria and downregulate other $\mathrm{Ca}^{2+}$ apoptosis-related genes to increase the sensitivity of $\mathrm{Ca}^{2+}$ and antitumoral activities. OSW-1 inhibits the VEGF/RTK/PI3K/AKT pathway to reduce angiogenesis and tumor growth in HCCs.

The p53 gene, as a tumor suppressor that is best characterized as a transcription factor which binds to specific DNA sequences, has a major role in the cell response to DNA damage. The BH3-only gene, Noxa, is a direct transcriptional target of p53 and has a role in DNA damage-induced p53-mediated apoptosis (15). OSW-1 transactivates and upregulates $\mathrm{BH} 3$, which is a member of the Bcl-2 family. Noxa has a significant affinity for p53 (16) and may be essential to apoptosis during cellular stress (17). P53 induces apoptosis by the transcriptional induction of Puma and Noxa, which encode the proapoptotic BH3-only Bcl-2 family of proteins. However, at the molecular level, the mechanisms of action of the Puma and Noxa proteins remain poorly defined. Although certain studies have previously hypothesized that p53 induces apoptosis largely via Puma (18), the present study observed that OSW-1 upregulated Noxa only and not Puma when inducing apoptosis in the hepatoma cells. Additionally, the apoptosis-associated targets of p53, PERP and Siah, were upregulated by OSW-1.

The Bcl-2 family members and the mitochondria are significant targets of p53 (19). Activated extensively in the p53 signaling pathway, p53 also alters the function of the mitochondria and subsequently mediates the release of cytochrome-c (20). Perforation of the mitochondrial membrane results in the release of several death-promoting factors, which ultimately neither caspase dependently or independently execute cell death (21). Even more noteworthy was the fact that caspase9 was downregulated in OSW-1 induced apoptosis in the present study. Also, FADD and Bid were expressed in the opposite manner to the classical apoptosis theory. Although mitochondria-regulated cell death program was activated, apoptosis induced by caspase was inhibited. In the extrinsic pathways, the cell-surface receptor Fas promotes cell death through caspase-8 (22). However, Fas appears to be dispensable in p53-dependent apoptosis (23). Therefore, OSW-1 had the ability to activate the extrinsic and intrinsic apoptotic pathways. At the same time, in the present study, this special type of mitochondrial apoptosis, which featured caspase inhibition induced by OSW-1, suggested that necroptosis was involved.

Necroptosis is a novel form of caspase-independent cell death. The initiation of programmed necrosis, necroptosis, by death receptors requires the kinase activity of receptor-interacting proteins and its execution involves the active disintegration of mitochondrial, lysosomal and plasma membranes (24). In a study by Han et al (25), necroptosis did not involve caspase, $\mathrm{Bcl}-2$ or the release of cytochrome-c from the mitochondria. Other previous studies $(9,26)$ are in agreement with the present study results which showed the release of cytochrome-c and the cleavage of Bcl-2 dependents by caspase 8 , OSW-1 target oxysterol-binding protein (OSBP) and its closest paralog, OSBP-related protein 4L (ORP4L). Additionally, FADD and caspase 9 were downregulated and the expression of RIPK2 was upregulated in the Hep3B OSW-1-treated cells. Of note was the fact that $\mathrm{BH} 3$ was required for death receptor-induced necroptosis (27) and that the characteristics of the upregulation of Noxa were expounded in the p53 pathway in the present study.

\section{Acknowledgements}

This study was financially supported by the National Natural Science Foundation of China (Grant No. 81160529) and the Jilin Province Science and Technology Development Project (grant no. 200905207)

\section{References}

1. Kubo S, Mimaki Y, Terao M, Sashida Y, Nikaido T and Ohmoto T: Acylated cholestane glycosides from the bulbs of Ornithogalum saudersiae. Phytochemistry 31: 3969-3973, 1992. 
2. Mimaki Y, Kuroda M, Kameyama A, Sashida Y, Hirano T and Oka K: Cholestane glycosides with potent cytostatic activities on various tumor cells from Ornithogalum saundersiae bulls. Bioorg Med Chem Lett. 7: 633-636, 1997.

3. Zhou Y, Garcia-Prieto C, Carney DA, et al: OSW-1: a natural compound with potent anticancer activity and a novel mechanism of action. J Natl Cancer Inst 97: 1781-1785, 2005.

4. Cai FG, Xiao JS and Ye QF: Effects of ischemic preconditioning on cyclinD1 expression during early ischemic reperfusion in rats. World J Gastroenterol 12: 2936-2940, 2006.

5. Kim EK and Choi EJ: Pathological roles of MAPK signaling pathways in human diseases. Biochim Biophys Acta 1802: 396-405, 2010.

6. McKay MM and Morrison DK: Integrating signals from RTKs to ERK/MAPK. Oncogene 26: 3113-3121, 2007.

7. Ballif, BA and Blenis J: Molecular mechanisms mediating mammalian mitogen-activated protein kinase (MAPK) kinase (MEK)-MAPK cell survival signals. Cell Growth Differ 12 397-408, 2001

8. Bonni A, Brunet A, West AE, Datta SR, Takasu MA and Greenberg ME: Cell survival promoted by the Ras-MAPK signalling pathway by transcription-dependent and -independent mechanisms. Science 286: 1358-1362, 1999.

9. Zhu J, Xiong L, Yu B and Wu J: Apoptosis induced by a new member of saponin family is mediated through caspase-8-dependent cleavage of Bcl-2. Mol Pharmacol 68: 1831-1838, 2005.

10. Porras A, Zuluaga S, Black E, et al: $\mathrm{P} 38$ alpha mitogen-activated protein kinase sensitizes cells to apoptosis induced by different stimuli. Mol Biol Cell 15: 922-933, 2004.

11. Grethe S and Pörn-Ares MI: p38 MAPK regulates phosphorylation of Bad via PP2A-dependent suppression of the MEK1/2-ERK1/2 survival pathway in TNF-alpha induced endothelial apoptosis. Cell Signal 18: 531-540, 2006.

12. Zhong Q, Zhou Y, Ye W, Cai T, Zhang X and Deng DY: Hypoxia-inducible factor 1- $\alpha$-AA-modified bone marrow stem cells protect PC12 cells from hypoxia-induced apoptosis, partially through VEGF/PI3K/Akt/FoxO1 pathway. Stem Cells Dev 21: 2703-2717, 2012

13. Adya R, Tan BK, Punn A, Chen J and Randeva HS: Visfatin induces human endothelial VEGF and MMP-2/9 production via MAPK and PI3K/Akt signalling pathways: novel insights into visfatin-induced angiogenesis. Cardiovasc Res 78: 356-365, 2008.
14. Chiang IT, Liu YC, Wang WH, et al: Sorafenib inhibits TPA-induced MMP-9 and VEGF expression via suppression of ERK/NF- $\mathrm{B}$ pathway in hepatocellular carcinoma cells. In Vivo 26: 671-681, 2012

15. Villunger A,MichalakEM,Coultas L, et al: p53-and drug-induced apoptotic responses mediated by $\mathrm{BH} 3$-only proteins puma and noxa. Science 302:1036-1038, 2003.

16. Park SY, Jeong MS and Jang SB: In vitro binding properties of tumor suppressor p53 with PUMA and NOXA. Biochem Biophys Res Commun 420: 350-356, 2012.

17. Huang DC and Strasser A: BH3-only proteins - essential initiators of apoptotic cell death. Cell 103: 839-842, 2000.

18. Michalak EM, Villunger A, Adams JM and Strasser A: In several cell types tumour suppressor p53 induces apoptosis largely via Puma but Noxa can contribute. Cell Death Differ 15: 1019-1029, 2008.

19. Vaseva AV and Moll UM: The mitochondrial p53 pathway. Biochim Biophys Acta 1787: 414-420, 2009.

20. Leu JI, Dumont P, Hafey M, Murphy ME and George DL: Mitochondrial p53 activates Bak and causes disruption of a Bak-Mcl1 complex. Nat Cell Biol 6: 443-450, 2004.

21. Gogvadze V, Orrenius S and Zhivotovsky B: Mitochondria as targets for cancer chemotherapy. Semin Cancer Biol 19: 57-66, 2009.

22. Nagata $S$ and Golstein P: The Fas death factor. Science 267: 1449-1456, 1995.

23. O'Connor L, Harris AW and Strasser A: CD95 (Fas/APO-1) and p53 signal apoptosis independently in diverse cell types. Cancer Res 60: 1217-1220, 2000.

24. Vandenabeele P, Galluzzi L, Vanden Berghe T and Kroemer G: Molecular mechanisms of necroptosis: an ordered cellular explosion. Nat Rev Mol Cell Biol 11: 700-714, 2010.

25. Han W, Li L, Qiu S, et al: Shikonin circumvents cancer drug resistance by induction of a necroptotic death. Mol Cancer Ther 6: 1641-1649, 2007.

26. Burgett AW, Poulsen TB, Wangkanont K, et al: Natural products reveal cancer cell dependence on oxysterol-binding proteins. Nat Chem Biol 7: 639-647, 2011.

27. Hitomi J, Christofferson DE, Ng A, Yao J, Degterev A, Xavier RJ and Yuan J: Identification of a molecular signalling network that regulates a cellular necrotic cell death pathway. Cell 135: 1311-1323, 2008. 